\title{
INTERVENÇÕES NA SOBRECARGA DE CUIDADORES INFORMAIS DE IDOSOS DEPENDENTES NA COMUNIDADE - REVISÃO INTEGRATIVA DA LITERATURA
}

OVERLOAD INTERVENTIONS FOR INFORMAL CAREGIVERS OF ELDERLY DEPENDENTS IN THE COMMUNITY - AN INTEGRATIIVE REVIEW OF THE LITERATURE INTERVENCIONES EN LA SOBRECARGA DE LOS CUIDADORES INFORMALES DE PERSONAS MAYORES DEPENDIENTES - REVISIÓN INTEGRATIVA DE LA LITERATURA EN LA COMUNIDAD

Verónica Lopes (veronica.lopes@hgo.min-saude.pt) * Helena do Arco (helenarco@ipportalegre.pt) **

\section{RESUMO}

Este estudo teve como objetivo realizar uma revisão integrativa da literatura, sobre intervenções para a prevenção da sobrecarga de cuidadores informais de idosos dependentes na comunidade, averiguando como poderia ser melhorado o papel dos enfermeiros, mobilizando a Teoria do Défice de Autocuidado de Enfermagem de Dorothea Orem. Efetuámos a pesquisa eletrónica de artigos na EBSCOhost e selecionámos nove, cujos resultados foram agrupados em três dimensões: I. Sobrecarga dos cuidadores de idosos; II. - Intervenções para cuidadores sustentadas no défice de autocuidado; e III. - Evidências sobre as intervenções de apoio a cuidadores informais. A implementação de intervenções multidisciplinares na comunidade, junto dos cuidadores informais, produz efeitos positivos na sobrecarga. As intervenções de enfermagem sustentadas na Teoria do Défice do Autocuidado de Enfermagem, particularmente no sistema de enfermagem educacional, evidenciam melhorias na saúde e no bem-estar dos cuidadores informais, reduzindo a sobrecarga subjetiva.

Palavras Chave: cuidadores, intervenções, sobrecarga.

\section{ABSTRACT}

This study aims to perform an integrative review of the literature on interventions in the work overload of informal caregivers of dependent elderly in the community, investigating how the role of nurses can be improved by mobilizing Dorothea Orem's Nursing Self-Care Deficit Theory. We performed the electronic research of articles in EBSCOhost, of which we selected nine and the results were grouped into three dimensions: I. - Overload of caregivers of the elderly; II. - Interventions for caregivers based on the self-care deficit; and III. - Evidence of interventions to support informal caregivers. The implementation of multidisciplinary interventions in the community, together with informal caregivers, has a positive effect on overload. Nursing interventions, supported by the Nursing Self- 
Care Deficit Theory, particularly in the educational nursing system, evidence improvements in the health and well-being of informal caregivers by reducing subjective overload.

Keywords: caregivers, overload, interventions.

\section{RESUMEN}

Este estudio tuvo como objetivo realizar una revisión integrativa de la literatura, sobre intervenciones para la prevención de la sobrecarga de cuidadores informales de ancianos dependientes en la comunidad, averiguando cómo puede mejorarse el papel de los enfermeros, movilizando la Teoría del Déficit de Autocuidado de Enfermería de Dorothea Orem. Hemos realizado la investigación electrónica de artículos en EBSCOhost, seleccionamos nueve, cuyos resultados se agruparon en tres dimensiones: I. - Sobrecarga de los cuidadores de ancianos; II. - Intervenciones para cuidadores sostenidos en el déficit de autocuidado; y III. - Evidencias sobre las intervenciones de apoyo a cuidadores informales. La implementación de intervenciones multidisciplinares en la comunidad, junto a los cuidadores informales, produce efectos positivos en la sobrecarga. Las intervenciones de enfermería, sostenidas en la Teoría del Déficit del Autocuidado de Enfermería, particularmente en el sistema de enfermería educativa, evidencian mejoras en la salud y el bienestar de los cuidadores informales, reduciendo la sobrecarga subjetiva.

Palabras clave: cuidadores, intervenciones, sobrecarga.

* RN - Serviço de Medicina II, do Hospital Garcia de Orta, Entidade Pública Empresarial, Almada, Portugal.

** PhD - Instituto Politécnico de Portalegre, Portugal; ESS, NURSE'INUIESI; CICS.NOVA.UÉvora.

Submitted: 28th February 2019

Accepted: 8th July 2019 


\section{INTRODUÇÃO}

Atualmente, no mundo, a maioria das pessoas pode esperar viver até aos 60 ou mais anos, de idade. Por seu lado, nos países de baixos e médios rendimentos, tal deve-se à redução das taxas de mortalidade nos jovens, principalmente na infância, relacionadas com o parto e por doenças infecciosas. No que concerne aos países de altos rendimentos, o aumento da esperança média de vida atribui-se, essencialmente, ao declínio da mortalidade dos mais idosos (Organização Mundial de Saúde [OMS], 2015). Dados da United Nations Economic Commission for Europe (UNECE, 2017) apontam que, em 2070, Portugal será o país com o maior rácio de dependência total $(89,7 \%)$, seguido pela Polónia $(86,7 \%)$, Grécia (86\%), Itália $(83,5 \%)$ e a Eslováquia (82,7\%). O número de idosos é superior ao número de jovens já há vários anos, sendo que o índice de envelhecimento atingiu os 157,4 idosos por cada 100 jovens, em 2018. Paralelamente, o índice de dependência, que nos informa sobre a relação entre o número de idosos e o número de pessoas em idade ativa ( 15 aos 64 anos), tem vindo a aumentar continuadamente nas últimas décadas, verificando-se em 2018, cerca de 33,6 idosos por cada 100 pessoas, em idade ativa. O envelhecimento da população decorre do aumento da longevidade e do declínio da fecundidade (Comissão Europeia, 2017). Dado que a percentagem de idosos é elevada ( $19 \%$ do total da população portuguesa), concentra uma maior prevalência de doenças crónicas, menor literacia geral e específica (de saúde), maior vulnerabilidade social, em associação com a debilidade das redes relacionais e financeira o que traduz um incremento do risco de pobreza, torna-se imperioso o desenvolvimento de respostas concertadas dos prestadores de cuidados de saúde (Observatório Português dos Sistemas de Saúde [OPSS], 2015). São os idosos quem mais utiliza os serviços de saúde, nas diferentes áreas de intervenção e tipologia (cuidados de saúde primários [consultas]; cuidados de saúde diferenciados [internamentos]; aquisição de medicamentos; etc.), quer por sofrerem de um número crescente de afeções proporcionais à idade quer pelo aumento da esperança média de vida (77,6 anos para as mulheres e 83,3 para os homens, em 2016). Esta procura elevada de cuidados tem conduzido a um crescimento contínuo das despesas, que ameaça a sustentabilidade do sistema de saúde português (PORDATA, 2018; Béresniak \& Duru 1999).

Ao aumento da longevidade estão associados maiores níveis de dependência, resultado da progressiva morbilidade e da maior vulnerabilidade dos idosos. As estruturas familiares sofreram grandes modificações nas últimas décadas, enfatizando o papel da mulher, enquanto cuidadora principal, em simultâneo com a acumulação de outros papéis, dificultando a prestação de cuidados. No contexto português, usualmente os cuidadores informais têm idade avançada (mais de 61 anos), são do género feminino (87\%), casados $(78,8 \%)$, com baixa escolaridade (somente $7,1 \%$ completaram o ensino superior). Predominam os cuidadores domésticos ou sem atividade, desempregados, reformados e com baixos rendimentos; maioritariamente cônjuges ou filhas (Sequeira, 2018).

O stresse crónico ocupacional associado ao impacto e sobrecarga associada aos cuidados, envolvendo um intenso contacto interpessoal, aumenta a propensão para o desenvolvimento da Síndrome de Burnout. Tal inclui três fatores importantes: exaustão emocional (falta de energia com exaustão); despersonalização (insensibilidade emocional) e a falta de envolvimento no local de trabalho. Esta síndrome é encarada com um problema psicossocial, que pode acarretar graves consequências (Marques, Landim, Collares \& Mesquita, 2011). A sobrecarga define-se como "a medida em que os cuidadores percecionam repercussões nas suas emoções, saúde física, vida social e situação financeira, como resultado de cuidar do seu familiar" (Zarit, Todd \& Zarit, 1986: 261). É comum encontrar-se na pesquisa, os termos de 
stresse e de sobrecarga associados, não obstante, são conceitos diferentes, cuja avaliação implica a aplicação de instrumentos de medida diferentes.

$\mathrm{O}$ apoio aos cuidadores na comunidade é fundamental, centrado em intervenções que visam melhorar a capacitação para a gestão dos cuidados e fornecer recursos, procurando reduzir a sobrecarga, stresse, ansiedade e depressão. As intervenções são geralmente programas de aconselhamento, educação e descanso (Broady \& Aggar, 2017). Os cuidadores informais, comummente familiares, cuidam diretamente de uma pessoa por vontade, necessidade ou obrigação cultural, sem remuneração e, muitas vezes, sem preparação prévia para tal, estando expostos a problemas relacionados com o cuidado, o que os torna vulneráveis e sujeitos alvo da intervenção de enfermagem, exigindo o desenvolvimento de estratégias de apoio e fortalecimento, na prevenção da sobrecarga (Martínez, Cardona \& Gómez-Ortega, 2016).

Embora, a revisão de literatura documente necessidades potenciais e reais de intervenção nos idosos dependentes e seus cuidadores informais, em Portugal, escassa investigação tem sido efetuada. Não obstante, em 2006, foi criada a Rede Nacional de Cuidados Continuados Integrados, da qual fazem parte as Equipas de Cuidados Continuados Integrados (ECCl), constituídas principalmente por enfermeiros, que prestam cuidados a pessoas com dependência na comunidade e desenvolvem a sua ação, essencialmente na população idosa, sem que se conheça o seu impacto na redução da sobrecarga do cuidador informal. Assim, entendemos pertinente o desenvolvimento de pesquisas, que estudem a eficácia dos diferentes programas de intervenção e, deste modo, possam contribuir para a melhoria da atuação dos enfermeiros na comunidade.

A escolha do suporte na Teoria do Défice do Autocuidado de Enfermagem [TDAE] para esta revisão, baseou-se na sua vasta amplitude para enquadrar a enfermagem, enquanto disciplina e profissão, permitindo a construção de narrativas acerca das intervenções dos enfermeiros, dos padrões de conhecimento em que se sustentam e que, em simultâneo, também constroem e melhoram, na busca de respostas para os problemas de saúde, de doença e de bem-estar das pessoas e populações a que prestam cuidados, como é o caso dos cuidadores informais e das pessoas cuidadas (Queirós, Vidinha \& Filho, 2014).

Este trabalho procura identificar as intervenções eficazes para a prevenção/redução da sobrecarga dos cuidadores informais, alicerçadas na TDAE, objetivando a melhoria da qualidade da intervenção de enfermagem na comunidade, no apoio aos cuidadores e às pessoas cuidadas. No sentido de orientar a pesquisa de informação, foi formulada a questão: Como podem os enfermeiros melhorar a sua intervenção na comunidade, ao nível da prevenção/redução da sobrecarga dos cuidadores informais? Na estruturação desta questão, recorremos à estratégia PICO (Joanna Briggs Institute [JBI], 2015): participantes; intervenção; contexto do estudo e resultados (outcomes).

\section{METODOLOGIA}

Uma revisão integrativa "é um método que tem como finalidade sintetizar resultados obtidos em pesquisas sobre um tema ou questão, de maneira sistemática, ordenada e abrangente. É denominada integrativa porque fornece informações mais amplas sobre um assunto/problema, constituindo, assim, um corpo de conhecimento" (Ercole, Melo \& Alcoforado, 2014: 13). Esta revisão segue as etapas preconizadas pelo Joanna Briggs Institute (JBI, 2015a), nomeadamente: formulação da questão para a elaboração da revisão integrativa da literatura; especificação dos métodos de seleção dos estudos; procedimento de extração dos dados; análise e avaliação dos estudos incluídos; extração dos dados e apresentação da 
revisão/síntese do conhecimento produzido e publicado. Realizou-se uma pesquisa na base de dados eletrónica (BD) Ebscohost (MEDLINE Complete, CINAHL Complete, Nursing \& Allied Health Collection: Comprehensive, MedicLatina e Cochrane Database of Systematic Reviews), utilizando os descritores DeCS (Descritores em Ciências da Saúde) nas línguas portuguesa, espanhola e inglesa: Cuidadores, Cuidadores, Caregivers; Sobrecarga, Sobrecarga, Burden; Intervenções, Intervenciones, Interventions, associando-se o operador booleano AND. Os seguintes critérios de inclusão foram estabelecidos: intervenções em cuidadores informais de adultos/idosos dependentes no domicílio, excluindo cuidadores informais de menores. Procurou-se por revisões sistemáticas, clínicas ou integrativas da literatura, experiências clínicas randomizadas ou controladas e experiências não-randomizadas, texto completo, com acesso gratuito, publicados entre 2013 e 2018. Apuraram-se 1038 artigos, pela pesquisa na base de dados. Os duplicados foram eliminados. Dos 1038 artigos, 974 foram excluídos após leitura de título e resumo, sendo que 64 artigos foram selecionados para análise completa. Catorze artigos foram incluídos para avaliação metodológica, determinando-se o nível de evidência (JBI, 2014). Posteriormente procedemos à aplicação das grelhas de avaliação crítica (JBI, 2017), sendo que apenas nove obtiveram resultados superiores a $75 \%$, e como tal foram incluídos nesta revisão, com sete referências de nível I, uma de nível II e uma de nível III. Os 9 artigos integrados nesta revisão cumprem todos os critérios de inclusão, abordam a temática da sobrecarga dos cuidadores informais e as intervenções na comunidade. O rumo e os resultados da pesquisa encontram-se explanados na Figura 1.

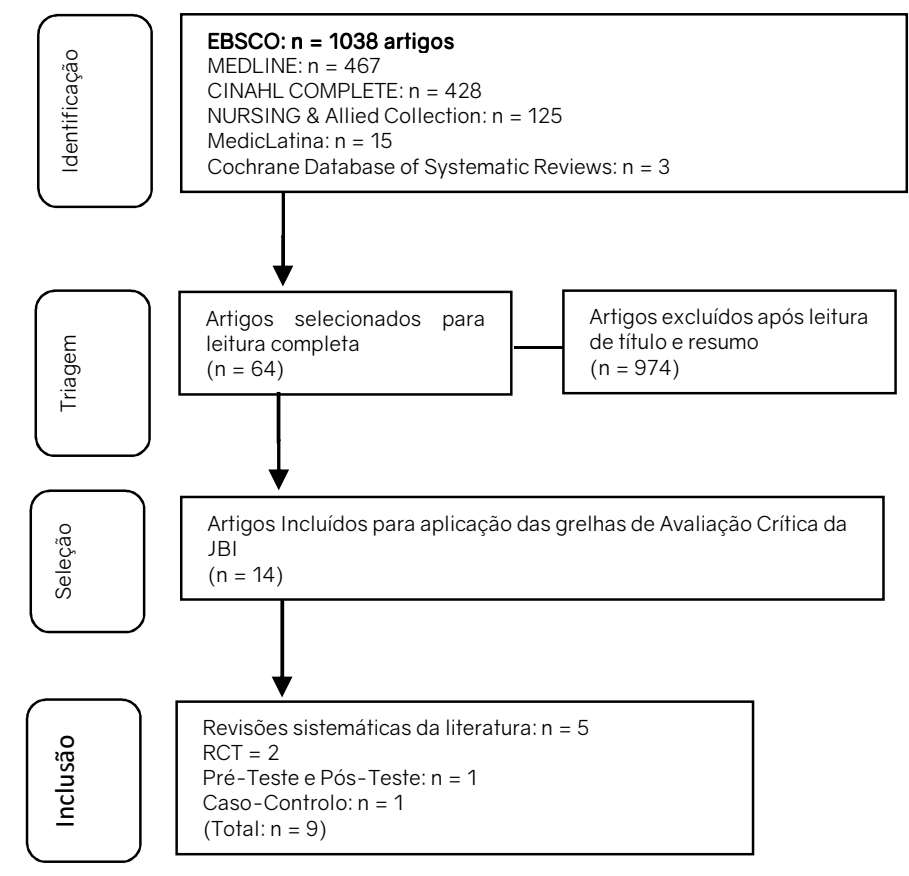

Figura 1: Fluxograma da Estratégia de Busca de Avaliação de Artigos 


\section{RESULTADOS}

Nove artigos foram incluídos nesta revisão, com sete referências de nível I, uma de nível II e uma de nível III (Quadro 1).

Os artigos analisados têm como tema principal, as intervenções na sobrecarga dos cuidadores informais na comunidade. Com a leitura dos trabalhos de investigação, percebemos que é um tema atual, relevante e bastante desafiador para os profissionais (frequentemente enfermeiros), que prestam apoio aos cuidadores e aos seus dependentes e que o conhecimento acerca das intervenções que se revelam mais eficazes, na redução do nível de exaustão, é essencial para introduzir melhorias na sua atuação.

Após a leitura e análise dos estudos, agrupámos os resultados em três dimensões: I. Sobrecarga dos cuidadores de idosos; II. - Intervenções para cuidadores sustentadas no défice de autocuidado; e III. - Evidências sobre as intervenções de apoio a cuidadores informais. A primeira dimensão está relacionada com a especial suscetibilidade à sobrecarga, dos cuidadores de idosos com demência. A segunda dimensão refere-se aos sistemas de enfermagem teorizados por Dorothea Orem e a sua utilização em benefício do autocuidado e bem-estar dos cuidadores. A terceira dimensão reporta-se, aos componentes das intervenções, que revelaram um impacto positivo na diminuição da sobrecarga. Em particular, no que respeita à nossa busca pelas intervenções eficazes, na redução da sobrecarga dos cuidadores informais, alicerçadas na TDAE, objetivando a melhoria da qualidade da intervenção de enfermagem na comunidade, no apoio aos cuidadores e às pessoas cuidadas, emergiram quatro categorias de intervenções: psicoeducacionais; psicoterapiaaconselhamento; multicomponente; e baseadas na tecnologia. No Quadro 2 apresentamos uma síntese dos artigos selecionados (ver Apêndice I).

\begin{tabular}{|c|c|}
\hline Autores, País e Ano de Publicação & Nível de Evidência \\
\hline $\begin{array}{l}\text { Kark, Graessel, Randzio \& Pendergrass. } \\
\text { Alemanha, } 2018{ }^{(a)}\end{array}$ & 3.d - Estudo Observacional, Caso-Controle \\
\hline $\begin{array}{l}\text { Bernal, Becerra \& Mojica. } \\
\text { Colômbia, } 2018^{(b)}\end{array}$ & $\begin{array}{l}\text { 2.d - Estudo Quasi-Experimental, Estudo Grupo de Controlo Pré- } \\
\text { Teste e Pós-Teste }\end{array}$ \\
\hline $\begin{array}{l}\text { Berwig, Heinrich, Spahlholz, Hallensleben, } \\
\text { Brähler \& Gertz. } \\
\text { Alemanha, } 2017 \text { (c) }\end{array}$ & 1.c - Estudo Experimental, RCT \\
\hline $\begin{array}{l}\text { Goeman, Renehan \& Koch. } \\
\text { Austrália, } 2016(2016)^{(d)}\end{array}$ & $\begin{array}{l}\text { 1.b - Estudo Experimental, Revisão Sistemática de RCT e outros } \\
\text { desenhos de estudo }\end{array}$ \\
\hline $\begin{array}{l}\text { Greenwood, Ferruccio, \& Hassenkamp. } \\
\text { Reino Unido, } 2016 \text { (e) }\end{array}$ & $\begin{array}{l}\text { 1.b - Estudo Experimental, Revisão Sistemática de RCT e outros } \\
\text { desenhos de estudogras }\end{array}$ \\
\hline $\begin{array}{l}\text { Chen, Huang, Yeh, Huang \& Chen. Japão, } 2015 \\
\text { (f) }\end{array}$ & 1.c - Estudo Experimental, RCT \\
\hline $\begin{array}{l}\text { Lins et al. } \\
\text { Alemanha, } 2014 \text { (g) }\end{array}$ & $\begin{array}{l}\text { 1.b - Estudo Experimental, Revisão Sistemática de RCT e outros } \\
\text { desenhos de estudo }\end{array}$ \\
\hline $\begin{array}{l}\text { Bunn, Goodman, Pinkney e Drennan. } \\
\text { Reino Unido, } 2014 \text { (h) }\end{array}$ & $\begin{array}{l}\text { 1.b - Estudo Experimental, Revisão Sistemática de RCT e outros } \\
\text { desenhos de estudo }\end{array}$ \\
\hline $\begin{array}{l}\text { Elvish, Lever, Johnstone, Cawley e Keady. } \\
\text { Reino Unido, } 2013^{(i)}\end{array}$ & $\begin{array}{l}\text { 1.b - Estudo Experimental, Revisão Sistemática de RCT e outros } \\
\text { desenhos de estudo }\end{array}$ \\
\hline
\end{tabular}

Quadro 1: Classificação dos Estudos por Nível de Evidência 


\section{DISCUSSÃO}

\subsection{Sobrecarga dos Cuidadores de Idosos}

Após a leitura e análise dos artigos selecionados, verificámos que oito dos nove estudos abordam a problemática da sobrecarga nos cuidadores informais, de pessoas com demência. A vulnerabilidade dos cuidadores de idosos com demência tem sido amplamente reconhecida. Com o aumento do índice de envelhecimento, aumenta também a probabilidade do aparecimento de doenças crónicas, incapacitantes e involutivas, comprometedoras da autonomia do indivíduo. A demência define-se como "uma síndrome que afeta o funcionamento normal do cérebro de natureza crónica e progressiva produzindo múltiplos deficits na memória, linguagem, raciocínio, julgamento, orientação e aprendizagem com intensidade suficiente para interferir no desempenho social do cidadão e/ou profissional" (Leite, Camacho, Queiroz, \& Feliciano, 2017: 889). A demência é a principal precursora de incapacidade nos idosos em todo o mundo e é a principal causa de dependência, stresse e sobrecarga do cuidador (Kim, Chang, Rose \& Kim, 2012; Maclennon, Habermann \& Rice, 2011).

Constatámos, uma maior sobrecarga subjetiva nos cuidadores informais de idosos com demência, o que é convergente com outras pesquisas. Estes dados demonstraram a importância de intervenções específicas, direcionadas aos cuidadores informais de pessoas com demência (Kark et al., 2018). Porém, o impacto do apoio de enfermeiros especialistas em demência "Admiral Nurses", na comunidade, é incerto, fruto da indefinição de resultados esperados, que possam paralelamente ajudar na organização dos serviços. Outras pesquisas devem focar-se, em avaliar os resultados atingidos por enfermeiros especializados em demência e outros profissionais (Bunn et al., 2014).

Apurámos igualmente, a carência de estudos acerca do impacto das intervenções de apoio para cuidadores informais de pessoas com acidente vascular cerebral (AVC) (Greenwood et al., 2014). Trata-se de uma grave lacuna, que deve ser considerada em investigações futuras, uma vez que o AVC (no ramo das doenças cardiovasculares) provoca uma elevada morbilidade e tem um impacto indiscutível na família e na sociedade, pelas sequelas associadas. Não obstante, a pesquisa de Silva et al. (2018) defende que as intervenções psicoeducativas, apoio e aquisição de habilidades têm implicações positivas quer para cuidadores (psicológicas, físicas, sociais, qualidade dos cuidados e aquisição de conhecimentos) quer para sobreviventes de AVC (menor utilização dos serviços de saúde e maior capacidade de autocuidado). As intervenções psicoeducacionais integram as componentes de apoio educacional e psicossocial, aumentando a perceção de autoeficácia dos cuidadores na gestão da doença e incrementando o desenvolvimento de estratégias de coping eficazes (Figueiredo, Guerra, Marques \& Sousa, 2012). A psicoeducação agrega uma vertente multidisciplinar, assinalando-se por ser circunscrita no tempo, estruturada em sessões, focada no presente e na técnica de resolução dos problemas, baseia-se sobretudo na literacia sobre a demência, nos sinais e sintomas, diagnóstico, etiologia, prognóstico, tratamentos farmacológicos e não farmacológicos, atraso da institucionalização e apoios sociais (Sousa, Mendes \& Relvas, 2007).

Deste modo, inferimos que os profissionais, nomeadamente os enfermeiros na comunidade, devem ter como uma das suas principais prioridades, a implementação de intervenções psicoeducacionais, junto dos cuidadores informais de idosos com demência, dadas as implicações na saúde e bem-estar dos mesmos. 


\subsection{Intervenções para cuidadores sustentadas no défice de autocuidado}

Verificámos que os cuidadores informais evidenciam dificuldades, na prestação de cuidados e na manutenção do bem-estar e que as intervenções de enfermagem, fundamentadas na teoria, favorecem os cuidados para o bem-estar dos cuidadores (Bernal et al., 2018; e Bunn et al., 2014). "O pensamento teórico tem evoluído num percurso de grande riqueza conceptual, gerando teorias e conceitos com valor interpretativo daquilo que os enfermeiros fazem." (Queirós, Vidinha \& Filho, 2014: 157). Ribeiro, Martins e Tronchin (2016:125) afirmam, igualmente, que "nos últimos anos, muitas organizações de saúde internacionais, têm valorizado os modelos de prática profissional de enfermagem como uma ferramenta para alcançar a excelência nos resultados dos clientes e dos enfermeiros". Por outro lado, para Söderhamn (2013: 13) "O autocuidado como conceito clínico é relevante para os profissionais de saúde, e deve ser significativo investigá-lo em nível filosófico e aprofundar ainda mais esse conceito".

A Teoria do Défice de Autocuidado de Enfermagem de Orem centra-se na conceção, de que a necessidade de cuidados de enfermagem está ligada à subjetividade da maturidade das pessoas, em relação às restrições da ação, relacionadas com a saúde ou com os cuidados de saúde, pelo que estas restrições as deixam total ou parcialmente incapazes de cuidar de si mesmas ou dos seus dependentes. Orem (2001) identificou três tipos de prática da ciência de enfermagem, nos sistemas de enfermagem, respetivamente: 1) sistema totalmente compensatório, substituição do indivíduo no autocuidado; 2) sistema parcialmente compensatório, assistência complementar do indivíduo no autocuidado e 3) sistema educacional, apoio-educativo, ensino e supervisão do indivíduo no autocuidado. Particularmente, o sistema de enfermagem parcialmente compensatório e o sistema educacional são os mais adequados, quando se pretende que a pessoa adquira conhecimentos e habilidades, no qual o enfermeiro assume o papel de regular a comunicação e o desenvolvimento de habilidades de autocuidado (Hernández, Pacheco, \& Larreynaga, 2017).

Em resumo, os cuidadores podem ter variações nas necessidades de autocuidado, nas capacidades individuais de satisfação das necessidades e nas necessidades de apoio e reconhecimento. Posto isto, consideramos imprescindível uma avaliação de enfermagem, que determine qual o grau de sobrecarga subjetiva e qual o défice no autocuidado, decorrentes da atividade de cuidar, que permita planear e implementar intervenções psicoeducacionais, essencialmente num sistema de enfermagem apoio-educativo, perspetivando aumentar a autoeficácia, autoestima, competência pessoal e habilidades para a tomada de decisão no contexto da prática de cuidados.

\subsection{Evidências sobre as intervenções de apoio a cuidadores informais}

Quanto às evidências, apurámos que a eficácia das intervenções de apoio aos cuidadores informais, na redução da sobrecarga, está associada à existência de vários componentes, nomeadamente: intervenção duradoura (6 a 12 meses); equipa multidisciplinar/interdisciplinar; colaboração interna para determinar necessidades reais; cooperação interprofissional; abordagem individualizada; profissionais especializados; acompanhamento contínuo (presencial e via telefónica); rede forte com conexões de proximidade e competências interpessoais (Goeman et al., 2016). Por outro lado, 
identificámos que as intervenções psicossociais e educativas podem ajudar os cuidadores a adotar estratégias de coping focadas em problemas e no suporte social, benéficas nas questões da diminuição da sobrecarga. Os fatores causadores de stresse ("stressors") podem conduzir a níveis significativos de sobrecarga no bem-estar e na qualidade de vida dos cuidadores. O coping entende-se como o processo de adaptação a uma situação de stresse, sendo evidente que os indivíduos em sobrecarga podem experienciar estados de angústia e de inadaptação. Cabe aos profissionais de saúde, ajudar os cuidadores a potenciar as suas habilidades de coping, apoiar as habilidades preexistentes e facilitar o desenvolvimento de novas. O método de coping pode ser classificado como positivo ou negativo: no coping positivo o cuidador faz um esforço ativo para gerir os "stressors", controlando ou alterando as circunstâncias dos cuidados e as respostas emocionais; no coping negativo o cuidador pode desvincular-se das circunstâncias do cuidado (Chen et al., 2015). O estudo de Pérez, Sánchez, Ocaña e Casado (2017) corrobora estes achados, sendo que teceram as seguintes conclusões: as estratégias de coping são importantes na avaliação de cuidadores informais, de idosos dependentes; a qualidade de vida dos cuidadores está relacionada com as suas estratégias de coping, a qualidade de vida pode ser agravada pelo coping do tipo evitamento e a sua qualidade de vida pode ser melhorada através do coping focado na emoção e no suporte social.

Em síntese, e para responder ao objetivo inicial desta revisão, identificámos quatro categorias de intervenções: psicoeducacionais (Bernal et al., 2018; Berwig et al., 2017; Goeman et al., 2016; Greenwood et al., 2016; Chen et al., 2015; Bunn et al., 2014; e Elvish et al., 2014); psicoterapia-aconselhamento (Bernal et al., 2018; Berwig et al., 2017; Goeman et al., 2016; Greenwood et al., 2016; Chen et al., 2015; Bunn et al., 2014; e Elvish et al., 2014) multicomponente (Berwig et al., 2017; Goeman et al., 2016; e Elvish et al., 2014) e baseadas na tecnologia (Bernal et al., 2018; Berwig et al., 2017; Goeman et al., 2016; Lins et al., 2014; e Elvish et al., 2014). As intervenções psicoeducacionais e psicoterapia-aconselhamento são as mais referenciadas, pelos autores dos estudos contidos nesta revisão. Nas intervenções psicoeducacionais e psicoterapia-aconselhamento, o profissional é o agente de mudança e de desenvolvimento social, emocional e comportamental dos cuidadores, fomentando a aquisição de conhecimentos e habilidades, que serão mobilizados para a prática de cuidados, dotando-os de estratégias de coping, comunicação e adaptação (Lemes \& Neto, 2017). De realçar, que as intervenções multicomponente (aconselhamento individual, trabalho em grupo de suporte e apoio telefónico) e as baseadas na tecnologia (telefone ou computador) são as mais eficazes (Elvish et al., 2013).

\section{CONCLUSÃO}

Procurando responder à questão inicial da pesquisa, como podem os enfermeiros melhorar a sua intervenção na comunidade, ao nível da sobrecarga dos cuidadores informais, através dos artigos analisados, concluímos que o desenvolvimento de intervenções centradas no cuidador é de extrema importância. Por conseguinte, é necessária uma avaliação do autocuidado e do bem-estar do cuidador, pois sem uma correta avaliação, que origine a formulação de um diagnóstico de enfermagem, indicativo da existência (ou ausência) de sobrecarga (e em que grau), não é possível planear intervenções que ajudem ou apoiem efetivamente os cuidadores. É necessário saber se o cuidado prestado afeta a capacidade do cuidador para cuidar de si próprio e/ou do seu dependente, e em que medida o afeta. É esse conhecimento que orienta a intervenção de enfermagem (sistema totalmente compensatório, sistema parcialmente compensatório e sistema educacional). A ausência de uma intervenção eficaz na redução da 
sobrecarga, pode resultar em consequências para a saúde, quer para o cuidador quer para a pessoa cuidada.

$\mathrm{Na}$ análise das diversas produções científicas, identificámos diferentes tipos de intervenções para cuidadores de idosos dependentes no domicílio, a desenvolver essencialmente por enfermeiros, psicólogos e técnicos de serviço social, que se enquadram nas seguintes categorias: psicoeducacionais, psicoterapia-aconselhamento; multicomponente e baseadas na tecnologia.

Um dos aspetos mais destacados nesta revisão refere-se à importância das intervenções psicossociais e educativas, com vista ao desenvolvimento estratégias de coping positivo, objetivando o envolvimento do cuidador, tornando-o capaz de controlar e modelar as situações de cuidados e adequar as respostas emocionais.

Vários estudos realçam igualmente a importância do apoio de profissionais de saúde, nomeadamente de enfermeiros, e do impacto das suas intervenções nas questões da sobrecarga, quer pelos cuidados que prestam diretamente aos idosos com dependência, quer pelo ensino, instrução e treino de competências promotoras da capacitação para a prestação de cuidados pelos cuidadores.

Apesar da dimensão dos estudos científicos integrados nesta revisão integrativa, podendo este particularismo ser considerado enquanto limitação, mas tendo em consideração que as intervenções evidenciadas são de certo modo comuns a todos os cuidadores em sobrecarga, pode dizer-se, sem menosprezar a individualidade de cada pessoa, que intervir no sistema de enfermagem educacional poderá contribuir para a redução da sobrecarga dos cuidadores.

Como recomendação, atendendo a que todos os estudos analisados foram realizados em países estrangeiros, sugerimos a realização de estudos randomizados ou controlados no nosso país, que busquem a eficácia das intervenções de enfermagem na comunidade, direcionadas à prevenção da sobrecarga de cuidadores de idosos dependentes. Eventualmente poderão ser efetuados incluindo cuidadores de idosos com sequelas de AVC ou com diagnóstico de demência, objetivando a pesquisa por evidências sobre as intervenções, consoante os níveis de sobrecarga percecionados pelos cuidadores.

\section{BIBLIOGRAFIA}

Base de Dados Portugal Contemporâneo (PORDATA). (2018). Números de Portugal. Quadroresumo. Disponivel em https://www.pordata.pt/Portugal/Quadro+Resumo/Portugal-230616;

Béresniak, A. \& Duru, G. (1999). Economia da Saúde. 1ạ Edição. Lisboa: CLIMEPSI EDITORES;

Bernal, N., Becerra, J. \& Mojica, C. (2018). Intervención de enfermería para el bienestar de cuidadores de personas en cuidado domiciliario. Revista Cuidarte, 9(1), 2045-2058. https://doi.org/10.15649/cuidarte.v9i1.479;

Berwig, M., Heinrich, S., Spahlholz, J., Hallensleben, N., Brähler, E. \& Gertz, H.-J. (2017). Individualized support for informal caregivers of people with dementia - effectiveness of the German adaptation of REACH II. BMC Geriatrics, 17(1), 286. https://doi.org/10.1186/s12877-017-0678-y;

Broady, T. \& Aggar, C. (2017). Carer interventions: an overview of service effectiveness. Jarna, 20 (2), 5-11;

Bunn, F., Goodman, C., Pinkney, E. \& Drennan, V. M. (2016). Specialist nursing and community support for the carers of people with dementia living at home: an evidence synthesis. Health \& Social Care In The Community, 24(1), 48-67. https://doi.org/10.1111/hsc.12189; 
Chen, H.-M., Huang, M.-F., Yeh, Y.-C., Huang, W.-H. \& Chen, C.-S. (2015). Effectiveness of coping strategies intervention on caregiver burden among caregivers of elderly patients with dementia. Psychogeriatrics: The Official Journal of The Japanese Psychogeriatric Society, 15(1), 20-25. https://doi.org/10.1111/psyg.12071;

Comissão Europeia. (2017). The 2018 Ageing Report - Underlying Assumptions \& Projecting Methodologies. Disponivel em: https://ec.europa.eu/info/sites/info/files/economyfinance/ip065_en.pdf;

Elvish, R., Lever, S.- J., Johnstone, J., Cawley, R. \& Keady, J. (2013). Psychological interventions for carers of people with dementia: A systematic review of quantitative and qualitative evidence. Counselling \& Psychotherapy Research, 13(2), 106-125. https://doi.org/10.1080/14733145.2012.739632;

Ercole, F., Melo, L. \& Alcoforado, C. (2014.). Revisão Integrativa versus Revisão Sistemática. Rev Min Enferm, 18 (1), 9-11;

Figueiredo, D., Guerra, S., Marques, A. \& Sousa, L. (2012). Apoio psicoeducativo a cuidadores familiares e formais de pessoas idosas com demência. Revista Temática Kairós Gerontologia, 15(1), 31-55. Disponível em https://revistas.pucsp.br/kairos/article/view/12776/9267;

Goeman, D., Renehan, E. \& Koch, S. (2016). What is the effectiveness of the support worker role for people with dementia and their carers? A systematic review. BMC Health Services Research, 16, 285. https://doi.org/10.1186/s12913-016-1531-2;

Greenwood, N., Pelone, F. \& Hassenkamp, A.-M. (2016). General practice based psychosocial interventions for supporting carers of people with dementia or stroke: a systematic review. BMC Family Practice, 17, 3. https://doi.org/10.1186/s12875-015-0399-2;

Hernández, Y., Pacheco, J. \& Larreynaga, M. (2017). La teoría Déficit de autocuidado: Dorothea Elizabeth Orem. Gaceta Médica Espirituana, 19(3), 7-17. Disponível em http://search.ebscohost.com/login. aspx?direct=true \&db=Ith\&AN=127975686\&lang=ptbr\&site = ehost-live;

JBI. (2015). Methodology for JBI Scoping Reviews. Disponível em http://joannabriggs.org/assets/docs/sumari/Reviewers-Manual_Methodology-for-JBI-ScopingReviews_2015_v2.pdf;

JBI. (2017). Critical Appraisal Tools. Disponível em http://joannabriggs.org/research/criticalappraisal-tools.html;

Joanna Briggs Institute (JBI). (2014). New JBI Levels of Evidence. Disponível em http://joannabriggs.org/assets/docs/approach/JBI-Levels-of-evidence_2014.pdf;

Karg, N., Graessel, E., Randzio, O. \& Pendergrass, A. (2018). Dementia as a predictor of care-related quality of life in informal caregivers: a cross-sectional study to investigate differences in healthrelated outcomes between dementia and non-dementia caregivers. BMC Geriatrics, 18(1), 189. https://doi.org/10.1186/s12877-018-0885-1;

Kim H., Chang M., Rose K. \& Kim S. (2012). Predictors of caregiver burden in caregivers of individuals with dementia. J Adv Nurs; 68: 846-855. https://doi.org/10.1111/j.1365-2648.2011.05787.x;

Leite, B., Camacho, A. C., Queiroz, R. \& Feliciano, G. (2017). Vulnerability of caregivers for the elderly with dementia: integrative review. Revista de Pesquisa: Cuidado e Fundamental, 9(3), 888-892. https://doi.org/10.9789/2175-5361.2017.v9i3.888-892;

Lemes, C.\& Neto, J. (2017). Aplicações da Psicoeducação no Contexto da Saúde. Trends in Psychology/Temas em Psicologia, 15(1), 17-28. Doi: 10.9788/TP2017.1-02. Disponível em http://pepsic.bvsalud.org/pdf/tp/v25n1/v25n1a02.pdf;

Lins, S., Hayder-Beichel, D., Rücker, G., Motschall, E., Antes, G., Meyer, G. \& Langer, G. (2014). Efficacy and experiences of telephone counselling for informal carers of people with dementia. The Cochrane Database Of Systematic Reviews, (9), CD009126. https://doi.org/10.1002/14651858.CD009126.pub2; 
Marques, A., Landim, F., Collares, P., \& de Mesquita, R. (2011). Social support in the family caregiver experience. Ciencia \& Saude Coletiva, 16 Suppl 1, 945-955. Disponível em http://search.ebscohost.com/login. aspx?direct=true \&db=mdc\&AN=21503442\&lang=pt-

br\&site=ehost-live;

Martínez, R. T., Cardona, E. M. \& Gómez-Ortega, O. R. (2016). Intervenciones De Enfermería Para Disminuir La Sobrecarga en Cuidadores: Un Estudio Piloto. Revista Cuidarte, 7(1), 1171-1184. https://doi.org/10.15649/cuidarte.v7i1.251;

McLennon SM., Habermann B. \& Rice M. (2011). Finding meaning as a mediator of burden on the health of caregivers of spouses with dementia. Aging Ment Health, 15: 522-530;

Observatório Português dos Sistemas de Saúde (OPSS). (2015). Acesso aos cuidados de Saúde. Um direito em Risco? - Relatório de Primavera 2015. Disponível em http://www.opss.pt/sites/opss.pt/files/RelatorioPrimavera2015.pdf;

Orem, D. (2001). Nursing: Concepts of practice. Saint Louis, MO, United States of America: Mosby;

Organização Mundial de Saúde (OMS). (2015). World report on Ageing and Health. Disponível em http://apps.who.int/iris/bitstream/handle/10665/186463/9789240694811_eng.pdf;jsessionid=F13 FE5D23533966FFE2014A20C9A0949? sequence =1;

Pérez, M., Sánchez, A., Ocaña, M. J. \& Casado, R. (2017). Coping strategies and quality of life in caregivers of dependent elderly relatives. Health and Quality of Life Outcomes, 15(1), 71. https://doi.org/10.1186/s12955-017-0634-8;

Queirós P., Vidinha, T. \& Filho, A. (2014). Autocuidado: o contributo teórico de Orem para a disciplina e profissão de Enfermagem. Revista de Enfermagem de Referência. Série IV (3), 157-164. Disponível em http://dx.doi.org/10.12707/RIV14081;

Ribeiro O., Martins M. \& Tronchin D. (2016). Modelos de prática profissional de enfermagem: revisão integrativa da literatura. Revista de Enfermagem Referência, IV (10), 125-133. http://dx.doi.org/10.12707/RIV16008;

Sequeira, C. (2018). Cuidar de Idosos com Dependência Física e Mental. 2.a Edição, Lisboa: Lidel;

Silva, J., Anjos K., Santos V., Boery R., Rosa D. \& Boery D. (2018). Intervenções para cuidadores de sobreviventes de acidente vascular cerebral: revisão sistemática. Rev Panam Salud Publica, 42, 1-9. https://doi.org/10.26633/RPSP.2018.114;

Söderhamn, O. (2013). Phenomenological perspectives on self-care in aging. Clin Interv Aging, 8 , 605-608. Doi: 10.2147/CIA.S45902;

Sousa, L., Mendes, A. \& Relvas, A. P. (2007). Enfrentara velhice e a doença crónica. Lisboa: Climepsi; United Nations Economic Commission for Europe (UNECE). (2017). Relatório de Portugal: Terceiro Ciclo de Revisão e Avaliação da Estratégia de Implementação Regional (RIS) do Plano Internacional de Ação de Madrid sobre o Envelhecimento (MIPAA). Disponível em https://www.unece.org/fileadmin/DAM/pau/age/country_rpts/2017/POR_report_POR.pdf;

Zarit, S., Todd, P.\& Zarit, J. (1986). Subjective Burden of Husbands and Wives as Caregivers: A Longitudinal Study. The Gerontological Society of America, 26 (3), 260-266. 
Quadro 2 - Artigos incluídos nesta Revisão Integrativa

\begin{tabular}{|c|c|c|c|}
\hline Título do Artigo & Objetivo & Metodologia e Amostra & Resultados/Conclusões \\
\hline $\begin{array}{l}\text { "Dementia as a predictor of care-related } \\
\text { quality of life in informal caregivers: a } \\
\text { cross-sectional study to investigate } \\
\text { differences in health-related outcomes } \\
\text { between dementia and non-dementia } \\
\text { caregivers"(a) }\end{array}$ & $\begin{array}{l}\text { Comparar os resultados relacionados coma } \\
\text { saúde, dos cuidadores informais de } \\
\text { pessoas com demência, com os dos } \\
\text { cuidadores que cuidam de familiares, com } \\
\text { outra doença crónica; } \\
\text { Verificar se a demência é um fator preditor } \\
\text { da qualidade de vida (QV) relacionada com } \\
\text { a prestação de cuidados, ao nível do } \\
\text { CarerQol-7D. }\end{array}$ & $\begin{array}{l}\text { Estudo transversal de dados auto- } \\
\text { relatados por } 386 \text { cuidadores informais, } \\
\text { que solicitaram avaliação ou re-avaliação } \\
\text { do nivel de cuidados, no Medical Service of } \\
\text { Compulsory Health Insurance Funds of } \\
\text { Bavaria. }\end{array}$ & $\begin{array}{l}\text { Os resultados demonstram diferenças significativas } \\
\text { entre os dois grupos de cuidadores em relação a } \\
\text { diferentes variáveis. Cuidadores informais de idosos } \\
\text { com demência relataram um nivel consideravelmente } \\
\text { elevado de sobrecarga subjetiva, maior do que no grupo } \\
\text { não-demência, que é consistente com outras pesquisas } \\
\text { sobre cuidados em demência e tensão percebida. } \\
\text { Os resultados do estudo sugerem que cuidar de um } \\
\text { familiar com demência está associado a pior saúde, ou } \\
\text { seja, maiores niveis de sobrecarga subjetiva e } \\
\text { depressão, e prediz menor QV em cuidadores informais. } \\
\text { Esses achados enfatizam a importância de intervenções } \\
\text { específicas com o objetivo de apoiar cuidadores } \\
\text { informais de pessoas com demência. }\end{array}$ \\
\hline $\begin{array}{l}\text { "Intervenção de enfermagem para o bem- } \\
\text { estar dos cuidadores das pessoas em } \\
\text { cuidados domiciliários"(b) }\end{array}$ & $\begin{array}{l}\text { Avaliar o impacto de uma intervenção de } \\
\text { enfermagem, no bem-estar do cuidador, de } \\
\text { pessoas com necessidades de cuidados no } \\
\text { domicílio, assistidos em instituições de } \\
\text { saúde de Boyacá, Colômbia. }\end{array}$ & $\begin{array}{l}\text { Estudo quasi-experimental, com pré-teste } \\
\text { e pós teste, com um grupo de } 72 \\
\text { cuidadores. O grupo de intervenção teve } \\
\text { três sessões educacionais, baseadas na } \\
\text { Teoria dos Cuidados de Swanson, o grupo } \\
\text { controle recebeu intervenção convencional. }\end{array}$ & $\begin{array}{l}\text { 1) O grupo de controlo evidenciou dificuldades no } \\
\text { cuidado do bem-estar, durante a prestação de cuidados; } \\
\text { 2) As intervenções de enfermagem, fundamentadas na } \\
\text { teoria, evidenciam melhorias nos cuidados para o bem- } \\
\text { estar das pessoas em condições especiais; } \\
\text { 3) É necessário estabelecer estratégias institucionais } \\
\text { focadas nos cuidados de enfermagem humanizados, } \\
\text { com sustentação disciplinar. }\end{array}$ \\
\hline $\begin{array}{l}\text { "Individualized support for informal } \\
\text { caregivers of people with dementia - } \\
\text { effectiveness of the German adaptation of } \\
\text { REACH II"(c) }\end{array}$ & $\begin{array}{l}\text { Traduzir e adaptar o programa REACH//,aos } \\
\text { requisitos do sistema de saúde alemão; } \\
\text { Avaliar a eficácia da adaptação alemã do } \\
R E A C H I I \text { (DE-REACH), centrada na } \\
\text { sobrecarga dos cuidadores informais de } \\
\text { pessoas com demência. }\end{array}$ & $\begin{array}{l}\text { Estudo controlado aleatório, com dois } \\
\text { grupos: experimental e de controlo. Do } \\
\text { grupo experimental (sujeito à intervenção) } \\
\text { faziam parte } 47 \text { cuidadores. O grupo de } \\
\text { controlo (sujeito a cuidados habituais) era } \\
\text { composto por } 45 \text { cuidadores. } \\
\text { A intervenção compreendeu } 12 \text { sessões } \\
\text { individuais, duas vezes por semana ( } 9 \text { no } \\
\text { domicilio com o cuidador informal e } 3 \text { via } \\
\text { telefone), e compreendeu cinco módulos. A } \\
\text { redução da sobrecarga dos cuidadores } \\
\text { informais foi escolhida como resultado } \\
\text { primário. }\end{array}$ & $\begin{array}{l}\text { Os resultados revelaram um grande efeito estabilizador } \\
\text { da intervenção na sobrecarga do cuidador, isto é, } \\
\text { comparando as medidas pré e pós, a sobrecarga } \\
\text { diminuiu muito ligeiramente, no grupo de intervenção, } \\
\text { aumentando significativamente no grupo de controlo. } \\
\text { Após um periodo de acompanhamento de três meses, } \\
\text { este efeito diminuiu de um efeito ótimo para moderado. } \\
\text { Verificaram-se também melhorias, como resultado da } \\
\text { intervenção na somatização, qualidade de vida } \\
\text { psicológica associada à saúde ea reação dos cuidadores } \\
\text { informais, em resposta a comportamentos do familiar } \\
\text { com demência. Paralelamente, a frequência de } \\
\text { comportamentos desafiadores da pessoa afetada em si } \\
\text { diminuiu durante a intervenção. } \\
\text { Os achados deste estudo fornecem mais evidências para } \\
\text { o impacto das intervenções de apoio multicomponentes, } \\
\text { para cuidadores informais de pessoas com demência. }\end{array}$ \\
\hline
\end{tabular}


"What is the effectiveness of the support worker role for people with dementia an their carers? A systematic review"(d)

"General practice based psychosocial interventions for supporting carers of people with dementia or stroke: systematic review"(e)

systematic review"(e)

"Effectiveness of coping strategies intervention on caregiver burden

among caregivers of elderly patients with dementia"(f)
Identificar os componentes principais e verificar como o papel dos profissionais pode ser melhor unilizado para ajudar as pessoas com demências e seus cuidadores.

Sintetizar as evidências disponíveis sobre $\mathrm{o}$ impacto das intervenções de apoio para cuidadores, desenvolvidas na prática clínica.

Promover o desenvolvimento de uma intervenção, direcionada à melhoria das estrategias de coping e analisar a sua na redução da sobrecarga do cuidador
Revisão sistemática de estudos entre Janeiro de 2003 e Dezembro de 2014. As fontes de pesquisa foram Medline, Psychinfo e CINAHL, internet, consulta especialistas e listas de referência de estudos incluídos.

A revisão sistemática seguiu as diretrizes PRISMA. A pesquisa foi efetuadas na seguintes bases de dados: MEDLINE;

EMBASE, Cochrane Library; PsycINFO; CINAHL Plus; Applied Social Sciences Index and Abstracts; Healthcare Management Information Consortium.

Estudo controlado aleatório, com dois grupos: experimental e de controlo. Do grupo experimental (sujeito à intervenção) faziam parte 24 cuidadores. 0 grupo de controlo (sujeito a cuidados habituais) composto por 22 cuidadores.
Componentes do suporte profissional:

1) A duração da intervenção deve ser de 6 a 12 meses, para existir um impacto significativo nas medidas da sobrecarga do cuidador, saúde em geral e bem-estar ou nas medidas da gravidade de sintomas da pessoa com demência;

2) Existir uma equipa multidisciplinar/interdisciplinar;

3) Existir uma colaboração interna para determinar qua - suporte necessário/providenciado (pessoa com demência, cuidador e familia);

4) Colaboração interprofissional e uma abordagem partilhada para cuidar;

5) Fornecer suporte individualizado para cada pessoa; 6) Garantir as competências especializadas dos profissionais de suporte (enfermeiro, terapeuta ocupacional, assistente social, treino em demências);

7) Providenciar um acompanhamento contínuo (visitas domiciliares a contacto telefónico) baseado nas necessidades;

8) Investir numa rede forte com conexões de proximidade com médicos/médico de família da pesso proxidância coordenando monitorizando o cuidado; 8) Capacidade de desenvolver relacionamentos. Auseria de estudos de avaliaça custo-efetiv

Não se averiguou qualquer investigação relacionada com intervençoes de apoio para cuidadores de pessoas com Acidente Vascular Cerebral. Os culdadores nâo emunerados desempenham um papel essencial, no cuidado às pessoas com Acidente Vascular Cerebral e Demencia. Não obstante, apurou-se uma escassez de investigaçoes acerca do impacto de intervenções de suporte para estes cuidadores. A evidencia disponivel sugere, que pode ser possivel oferecer apoio a esses prestadores de cuidados, na prática geral, porem, as pesquisas futuras devem centrar-se nas mesmas medidas de resultados, a fim de possibilitar comparações entre intervenções.

Os cuidadores alocados ao grupo experimental apresentaram redução na sobrecarga. As intervencões psicossociais e educativas podem ajudar os cuidadores a adotar estratégias de coping focadas em problemas no suporte social benéficas nas questões da diminuicão da sobrecarga. Apenas as estratégias de coping da sobrecarga. Apenas as estrateglas de coping 


\begin{tabular}{|c|c|c|c|}
\hline & & & $\begin{array}{l}\text { consequentemente, a sobrecarga do cuidador foi } \\
\text { reduzida. }\end{array}$ \\
\hline $\begin{array}{l}\text { "Efficacy and experiences of telephone } \\
\text { counselling for informal carers of people } \\
\text { with dementia (Review)"(g) }\end{array}$ & $\begin{array}{l}\text { 1) Produzir uma revisão quantitativa acerca } \\
\text { da eficácia do aconselhamento por telefone } \\
\text { para cuidadores informais de pessoas com } \\
\text { demência; } \\
\text { 2) Sintetizar estudos qualitativos, } \\
\text { explorando as experiências dos } \\
\text { cuidadores, na receção do aconselhamento } \\
\text { telefónico e as experiências dos } \\
\text { conselheiros, na realização de } \\
\text { aconselhamento por telefone; } \\
\text { 3) Integrar 1) e 2), para identificar aspetos } \\
\text { da intervenção, que são valorizados e } \\
\text { funcionam bem, assim como, os } \\
\text { componentes desta, que devem ser } \\
\text { melhorados ou redesenhados. }\end{array}$ & $\begin{array}{l}\text { Revisão de estudos controlados aleatórios, } \\
\text { que individualmente ou em grupos } \\
\text { compararam o aconselhamento por } \\
\text { telefone para cuidadores, de pessoas com } \\
\text { demência sem tratamento, cuidados } \\
\text { habituais ou contactos amigáveis para } \\
\text { conversas. Também foram considerados } \\
\text { estudos cross-over. }\end{array}$ & $\begin{array}{l}\text { Resultados primários: sintomas depressivos. Resultados } \\
\text { secundários: sobrecarga do cuidador; angústia; } \\
\text { ansiedade; qualidade de vida; autoeficácia do cuidador } \\
\text { e satisfação. } \\
\text { A análise de ambos os conjuntos de resultados, ou seja, } \\
\text { a eficácia comparada com a informação sobre as } \\
\text { experiências dos prestadores de cuidados por telefone, } \\
\text { revelou necessidades não atendidas pelo } \\
\text { aconselhamento telefónico. Os estudos que examinaram } \\
\text { o aspeto da experiência cobriram um número muito } \\
\text { limitado de tipos de aconselhamento por telefone. Os } \\
\text { resultados desta revisão devem ser interpretados com } \\
\text { cautela devido ao pequeno número de estudos e com } \\
\text { qualidade moderada. }\end{array}$ \\
\hline $\begin{array}{l}\text { "Specialist nursing and community support } \\
\text { for the carers of people with } \\
\text { dementia living at home: an evidence } \\
\text { synthesis"(h) }\end{array}$ & $\begin{array}{l}\text { Resumir a literatura acerca da eficácia dos } \\
\text { enfermeiros especialistas -"Admiral } \\
\text { Nurses", e definir esta evidência, no } \\
\text { contexto de outras iniciativas, em } \\
\text { comunidades, para apoiar os cuidadores } \\
\text { familiares de pessoas com demência. }\end{array}$ & $\begin{array}{l}\text { A síntese das evidências foi realizada em } \\
\text { duas fases: a fase } 1 \text { envolveu uma revisão } \\
\text { sistemática da literatura relacionada com o } \\
\text { papel de "Admiral Nurse" e a fase } 2 \text { que } \\
\text { compreendeu uma revisão sobre as } \\
\text { intervenções de apoio para cuidadores } \\
\text { familiares de pessoas com demência, na } \\
\text { comunidade. }\end{array}$ & $\begin{array}{l}\text { Há evidências de que o apoio, na comunidade, para } \\
\text { cuidadores de pessoas com demência (como ofornecido } \\
\text { por "Admiral Nurses") seja valorizado por cuidadores } \\
\text { familiares. O impacto de tais iniciativas ainda está por } \\
\text { estabelecer, sendo necessário definir quais os } \\
\text { resultados que podem ajudar a organizar a prestação de } \\
\text { serviços, para esta população, ao longo do tempo. Há } \\
\text { também a necessidade, de uma estrutura compartilhada } \\
\text { que possa informar como são definidas, } \\
\text { operacionalizadas e avaliadas as metas dos serviços. } \\
\text { Outras pesquisas devem focar-se em identificar o que } \\
\text { devem atingir (razoavelmente) os enfermeiros } \\
\text { especializados em demência e outros profissionais, em } \\
\text { diferentes fases da trajetória da demência e quais os } \\
\text { aspetos do seu papel que se revelam mais eficazes. }\end{array}$ \\
\hline $\begin{array}{l}\text { "Psychological interventions for carers of } \\
\text { people with dementia: } \\
\text { A systematic review of quantitative and } \\
\text { qualitative evidence"(i) }\end{array}$ & $\begin{array}{l}\text { Sintetizar as evidências disponíveis, acerca } \\
\text { das intervenções psicológicas, destinadas } \\
\text { a cuidadores de pessoas com demências. }\end{array}$ & $\begin{array}{l}\text { Revisão sistemática de estudos } \\
\text { quantitativos e qualitativos, publicados } \\
\text { entre } 2005 \text { e } 2011 \text {. As fontes de pesquisa } \\
\text { foram as bases de dados eletrónicas: } \\
\text { Medline, PsycINFO, ERIC e PubMed. }\end{array}$ & $\begin{array}{l}\text { Identificaram-se três categorias de intervenção } \\
\text { psicológica (consistentes com pesquisas anteriores): } \\
\text { 1) construção de habilidades psicoeducacionais; } \\
\text { 2) psicoterapia-aconselhamento; } \\
\text { 3) multicomponente. } \\
\text { Apurou-se uma quarta categoria (nesta revisão): } \\
\text { 4) baseadas na tecnologia. } \\
\text { A maioria dos estudos analisa a depressão, a } \\
\text { sobrecarga, o apoio social e o bem-estar. O } \\
\text { desenvolvimento de intervenções focadas nos } \\
\text { cuidadores, que sejam individuais ou em grupo, foi } \\
\text { significativo para a prática. }\end{array}$ \\
\hline
\end{tabular}


